\title{
Acute Bilateral Simultaneous Optic Neuritis
}

\author{
Charles E. Maxner,' MD, FRCPC and Kevin M. Ramsey, ${ }^{2} M D$ \\ 'Departments of Medicine (Neurology) and Ophthalmology, QEII Health Sciences Centre, Dalhousie \\ University, Halifax, NS \\ ${ }^{2}$ First year resident, Neurology, Faculty of Medicine, Dalhousie University
}

Background: Acute bilateral simultaneous optic neuritis (ABSON) without a prior neurological history is a rare clinical problem, and little is known about its prognosis for visual recovery and for the development of multiple sclerosis (MS). Methods: We conducted a case review of six patients who presented with ABSON from 1986-98, and did a follow-up on their present medical status. The results were compared to similar case reviews, and to the unilateral Optic Neuritis Treatment Trial. Results: All six patients went on to recover normal vision. One of the six patients $(16.7 \%)$ reviewed went on to develop probable MS. Interpretation: This low association with MS is in agreement with other case reports of ABSON. ABSON appears to have a significantly better prognosis for not developing neurological disease than unilateral optic neuritis. In particular, patients with ABSON without associated systemic symptoms tend to recover quickly to normal health.

\section{INTRODUCTION}

The prognostic value of unilateral optic neuritis has been well documented. ${ }^{1,2}$ Bilateral simultaneous optic neuritis in adults is significantly less common and consequently less information is available concerning visual recovery and its role as an indicator for the subsequent development of multiple sclerosis (MS). The focus of this study was to conduct a retrospective review of the cases of acute bilateral simultaneous optic neuritis (ABSON) that presented to the neuro-ophthalmology service at the QEII Health Sciences Centre in Halifax from 1986 to 1998 . The outcome for visual and neurological disease was recorded and compared to the prognostic results of similar ABSON studies ${ }^{3,4}$ and the unilateral Optic Neuritis Treatment Trial (ONTT). ${ }^{1,2}$

\section{METHODS}

Patients were identified retrospectively by searching hospital and service records. Patients were identified with an acute clinical syndrome consistent with optic neuritis in both eyes, where the start of visual symptoms in both eyes occurred within less than one week of each other. Patients under 18 years old, or with a previous neurological history were excluded. A systematic review of the patients' in-hospital
Dr. K. M. Ramsey, Box 82, Tantallon, Halifax, N.S., BOJ 3J0 records and follow-up contact with the patient or their family doctor was conducted.

Normal vision was defined as a visual acuity (VA) of $6 / 12$ or better in both eyes. Patients were given a diagnosis of probable multiple sclerosis (MS) based on a clinical evaluation consistent with MS without a known alternate cause and without positive radiological confirmation. Definite MS was defined by a clinical evaluation consistent with MS together with positive radiological confirmation.

Six patients were identified through the record search. The age of the patients at initial onset of symptoms ranged from 29 to 52 years of age. Follow-up was achieved in all patients for a minimum of five years except for one (Case 6) who was followed for only nine months.

A Medline search was performed to locate other studies of ABSON and the results of the ONTT. Our own data was then compared to these studies.

\section{RESULTS}

All six patients presented with vision loss, abnormal visual fields and bilateral disc edema. All six patients proceeded to recover to normal vision within six months of onset. A summary of the data is presented in Table 1 .

The six patients could be easily di- 
Table 1. ABSON Case Presentations

\begin{tabular}{|ccccccc|}
\hline Cases & Prior URTI & Headache & VF Defects & Other Symptoms $^{2}$ & Relapse $^{\text {Final Diagnosis }}$ \\
\hline 1 & 1 wk & + & + & - & - & Bilateral Papillitis \\
2 & 4 wks & + & + & - & - & Bilateral Papillitis \\
3 & 3 wks & + & + & + & - & Bilateral Papillitis \\
4 & - & + & + & + & - & Devic's Syndrome \\
5 & - & + & + & + & + & SLE \\
6 & - & + & + & + & + & Probable MS \\
\hline
\end{tabular}

"Other than visual abnormalities or headache

vided into two broad categories. The first group (Cases 1-3) presented with a prior history of upper respiratory tract infection (URTI), with classical symptoms of bilateral optic neuritis and without any other associated symptoms. These patients went on to recover very rapidly (within two weeks) to normal vision and did not suffer any relapses. The other group (Cases 4-6) did not present with a prior history of an URTI, but did present with the symptoms of optic neuritis in association with other systemic, and in particular, neurological symptoms. Prior URTI may suggest a more benign etiology of the optic neuritis, while more severe symptomatology may suggest a more severe disease process. Two of the three patients (Cases 5 and 6) suffered repeated relapses in the first four months before recovering normal vision. In addition, these two patients continued to suffer from repeated attacks of their systemic symptoms. The following case reports are examples of each group and illustrate the differences between the case presentations.

\section{Case Reports}

Case 2: A 44 year old female presented with vision reduced to finger counting in both eyes, and abnormal visual fields (loss of inferior hemifield and progressive loss of superior hemifield on the right [OD], marked involvement of superior hemifield on the left [OS]). Vision loss began with central vision dimming $\mathrm{OD}$ which continued to deteriorate, followed by similar involvement $\mathrm{OS}$ one day later. A relative afferent pupillary defect was observed in the right eye. Bilateral disc edema was noted on fundoscopy. The patient had developed a viral URTI one month prior to ocular symptoms, and at two weeks prior developed retroorbital headache with sharp pain on eye movement. The patient was treated with intravenous (IV) methylprednisolone (starting at $500 \mathrm{mg} /$ day) for five days followed by a ten day oral prednisone taper (starting dose at $60 \mathrm{mg} /$ day). At three week follow-up the patient had returned to normal vision (VA 6/6 OD, 6/4.5 OS). She was given a diagnosis of Bilateral Papillitis.

Case 6: A 29 year old female presented with gradual vision loss in both eyes to no light perception over three days and decreased pin prick sensation in her lower left limb. She was treated with intravenous methylprednisolone (starting at 1000 $\mathrm{mg} /$ day) for five days followed by an oral prednisone taper (starting dose at $60 \mathrm{mg} /$ day) and her vision recovered. Her MRI scan was negative except for positive signs of optic neuritis (no demyelinating lesions). At three weeks follow-up, she complained of pain on eye movement, fatigue, and paresthesia in her legs from the hips down. Vision was $6 / 7.5$ $\mathrm{OD}, 6 / 6 \mathrm{OS}$. At five weeks follow-up, she had a relapse of her ocular symptoms in both eyes with fatigue, headache, and paresthesia in her legs. She was treated with oral prednisone (starting dose at $60 \mathrm{mg} /$ day). Less than a week later, she again developed paresthesia in both lower extremities from midthigh distal, sphincter problems and fatigue. Visual acuity was $6 / 18$ OD, 6/7.5 OS with abnormal visual fields. Two weeks later the patient developed an URTI which coincided with an exacerbation of ocular and systemic symptoms. Bilateral temporal optic disc pallor was noted on fundoscopy. At followup 2.5 months after the most recent exacerbation, the patient complained of discomfort and numbness in lower extremities. Visual acuity was 6/6 OD, 6/7.5-1 OS with normal visual fields. She had not experienced any further visual problems in six months follow-up since the last visual relapse. However, at eight month follow-up she still continued to suffer from her systemic symptoms. She was given a diagnosis of probable MS.

\section{DISCUSSION}

Acute Bilateral Simultaneous Optic Neuritis rarely occurs without a previous history of neurological symptoms such as a demyelinating disease. This study reviewed only those cases of ABSON which did not have a previous neurological history. Six patients were identified. There were more females (five) than males (one).' A comparison of data between this review (Halifax Review), other ABSON reviews and the ONTT is listed in Table 2.

\section{Multiple Sclerosis}

Only one of the six patients (Case 6) was given a diagnosis of MS within a five-year follow-up. The other five patients did not have a recurrence of abnormal neurological symptoms. Four of the six patients underwent MRI scan (cases 3,4,5,6) and all had normal findings (no demyelinating lesions).

The case reports were similar to other acute bilateral simultaneous optic neuritis case series. A British study by Parkin et al. ${ }^{3}$ conducted an up to 37-year follow-up on six patients. Only one of their six patients was given a diagnosis of possible-probable MS of the benign type. This diagnosis was given on the basis of a telephone interview many years after her optic neuritis. The other five patients were free of other neurological symptoms.

A Greek case series by Moschos ${ }^{4}$ also was similar to this review. Moschos only followed those patients who presented with ABSON without other systemic symptoms. None of his six subjects developed signs of neurological disease by 
Table 2. Comparison of Optic Neuritis Studies

\begin{tabular}{|c|c|c|}
\hline \multicolumn{3}{|c|}{ Recovery of Normal Vision ${ }^{a}$ Possible/Probable/Definite MS } \\
\hline Bilateral Simultaneous Optic Neuritis: combined reviews & $89 \%(16 / 18)^{b}$ & $11 \%(2 / 18)$ \\
\hline Halifax Review & $100 \%(6 / 6)$ & $17 \%(1 / 6)$ \\
\hline Parkin Review ${ }^{3}$ & $67 \%(4 / 6)^{b}$ & $17 \%(1 / 6)$ \\
\hline Moschos Review ${ }^{4}$ & $100 \%(6 / 6)$ & $0 \% \quad(0 / 6)$ \\
\hline Unilateral Optic Neuritis & & \\
\hline ONTT $^{1,2}$ & $93.3 \%(409 / 438)$ & $45.6 \%(207 / 454)^{c}$ \\
\hline
\end{tabular}

the end of a three-year follow-up.

However, the results differ from those obtained from unilateral optic neuritis studies. In the ONTT $^{2}, 46 \%$ of the trial patients were given a clinical diagnosis of either probable or definite MS at the end of five-year follow-up. However upon initial assessment at entry to the study, $14.2 \%$ were listed as having probable-definite MS. This significantly high association is in contrast to the low association found in the preceding bilateral case reviews.

\section{Visual Recovery}

All six patients went on to recover normal vision $(6 / 12$ or better) by six months. Two of the six patients (Cases 5 and 6 ) suffered multiple relapses within the first four months, while the other four recovered within three weeks.

In the study by Parkin et al. ${ }^{3}$ two of the six patients had decreased vision at follow-up. However, it should be noted that this review was conducted up to 37 years after initial symptoms of optic neuritis at which time the subjects were quite elderly and may have lost their vision through other processes unrelated to their optic neuritis.

Moschos ${ }^{4}$ noted that all six of his patients recovered $6 / 6$ vision within one week of onset. None had experienced any visual disturbances after a three-year follow-up.

The ONTT ${ }^{1}$ also experienced a high rate of visual recovery after unilateral optic neuritis. At 6-month followup, $93.3 \%$ of patients recovered normal vision.

\section{Differentiating ABSON Cases}

The typical ABSON patient presents with sudden, severe vision loss in both eyes. Those without other systemic symptoms (Cases 1-3) appear to have a rapid recovery to normal health. Those ABSON patients that present with a more complicated picture (Cases 4-6) tend to have a more variable outcome, ranging from a rapid and full recovery to a severe systemic debilitating disease.

\section{SUMMARY}

While acknowledging the limited number of study patients in this case series and in the literature, the evidence seems to indicate that acute bilateral simultaneous optic neuritis has a better prognosis than unilateral optic neuritis for not developing MS. Its prognosis for full recovery of visual function af- ter 6 months appears to be very good.

\section{REFERENCES}

1. Beck RW et al. The Course of Visual Recovery after Optic Neuritis. Ophthalmology 1994; 101(11):1771-78.

2. Optic Neuritis Study Group. The 5-year Risk of MS after Optic Neuritis. Neurology 1997; 49(5): 1404-13.

3. Parkin PJ, Hierons R, McDonald WI. Bilateral Optic Neuritis A Long Term Follow-Up. Brain 1984;107:951-964.

4. Moschos M. Acute Bilateral Optic Neuritis. Doc Ophthalmol. 1989; 79(3):225-30. 


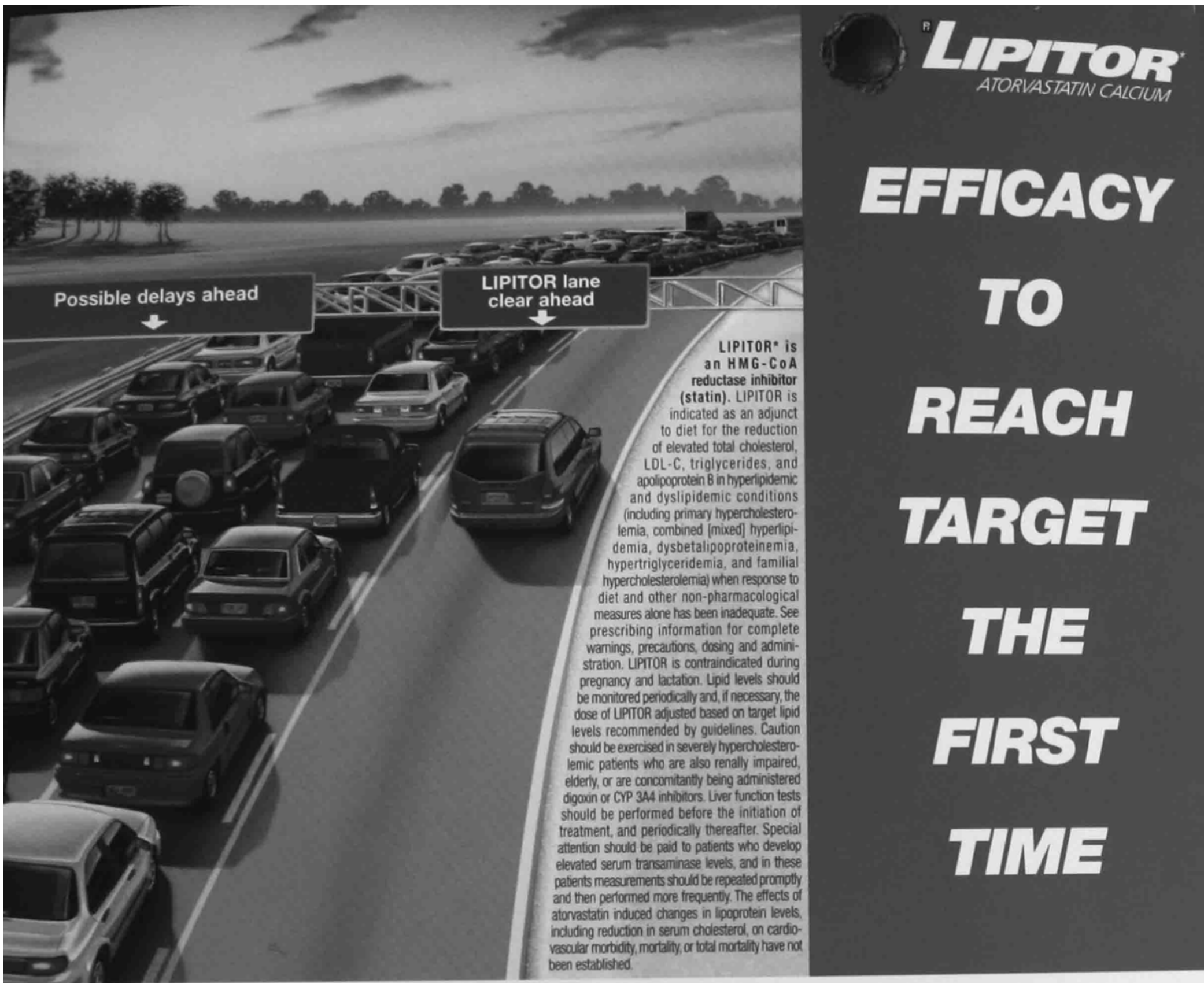

Recent clinical data showed that LIPITOR actually gets patients to target with fewer titrations and fewer repeat visits than ${ }^{{ }^{P} Z^{2}}$ ocor $^{\circ}$ (simvastatin),

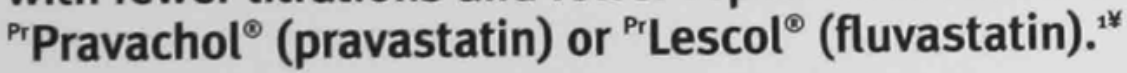

- Exceptional LDL-C reductions of $39-60 \%$ (Type Ila and IIb) over the full dose range ${ }^{2}$

- Significantly better LDL-C and TC/HDL-C ratio reductions compared to Zocor or Pravachol at starting doses ${ }^{3.45 t}$

- The added benefit of excellent TG reductions of 25-56\% (Type IV) over the full dose range $\mathrm{e}^{2}$

- Competitive price - LIPITOR costs less than Zocor or Pravachol at usual starting dose s $^{5}$

- Less than $2 \%$ of patients discontinued therapy due to adverse experiences. Most common adverse effects were myalgia, headache, constipation, diarrhea, dyspepsia, flatulence and nausea 